Z Gerontol Geriat 2022 · 55:136-142 https://doi.org/10.1007/s00391-022-02026-6 Eingegangen: 27. Mai 2021

Angenommen: 13. Januar 2022

Online publiziert: 15 . Februar 2022

(c) Der/die Autor(en) 2022

\section{Belastungserleben pflegender Angehöriger während der Coronapandemie}

\author{
Christina Theurer ${ }^{1}$. Doreen Rother ${ }^{1} \cdot$ Klaus Pfeiffer $^{2} \cdot$ Gabriele Wilz $^{1}$ \\ ${ }^{1}$ Institut der Psychologie, Abteilung Klinisch-Psychologische Intervention, Friedrich-Schiller-Universität \\ Jena, Jena, Deutschland \\ ${ }^{2}$ Robert-Bosch-Krankenhaus, Stuttgart, Deutschland
}

\title{
Zusammenfassung
}

Hintergrund: Bereits vor der Coronapandemie waren pflegende Angehörige mit einer Vielzahl an Herausforderungen und Belastungen konfrontiert. Erste Onlineerhebungen zeigten eine Zunahme der Pflegebelastung während der Pandemie. Ergänzend hierzu wurde eine Erhebung zu Auswirkungen der Pandemie auf bereits belastete pflegende Angehörige in Deutschland durchgeführt.

Methode: Im Rahmen einer Evaluationsstudie zu einem gestuften Beratungsansatz für hochbelastete pflegende Angehörige wurden von 165 Pflegenden quantitative und qualitative Daten zum Belastungserleben, zu Ängsten und Wünschen in der Coronapandemie bezüglich der Pflege erhoben. Die Auswertungen erfolgten deskriptiv und mittels qualitativer Inhaltsanalyse.

Ergebnisse: Von den Angehörigen gaben $26 \%$ starke Ängste an, sich mit SARSCoV-2 zu infizieren, $50 \%$ befürchteten, dass die gepflegte Person erkranken könnte. Die Hälfte berichtete deutliche Auswirkungen auf den Pflegealltag (47\%) und nahm eine deutliche Erhöhung der Pflegbelastung (51\%) wahr. Als häufigste negative Auswirkungen auf den Pflegealltag wurden der Ausfall von Pflegedienstleistungen und ein Mangel an Zeit für sich selbst genannt. Dementsprechend wurde am häufigsten der Wunsch nach Unterstützung geäußert. Der Pflegegrad, das Alter der Pflegenden und die vorherige Nutzung des Pflegedienstes erwiesen sich als Prädiktoren für das durch die Pflegenden eingeschätzte Belastungserleben der Erkrankten.

Diskussion: Die Ergebnisse verdeutlichen die negativen Auswirkungen der Coronapandemie auf die Belastung pflegender Angehöriger eindrücklich. Zur Bewältigung der komplexen Zusatzbelastungen benötigen Angehörige Angebote, die zu deren Teilhabechancen sowie zur Verbesserung der Versorgung und sozialen Teilhabe der Pflegebedürftigen während der Pandemie beitragen.

\section{Schlüsselwörter}

Informelle Pflege · Pflegebelastung · Psychosoziale Belastung · SARS-CoV-2 · COVID-19

\section{Studienregistrierung}

German Clinical Trials Register, DRKS00014593 (www.drks. de/DRKS00014593) und International Clinical Trials Registry Platform, DRKS00014593 (https://apps.who.int/ trialsearch/)

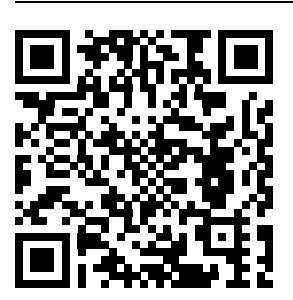

QR-Code scannen \& Beitrag online lesen

\section{Hintergrund}

Aktuell werden von den 4,1 Mio. pflegebedürftigen Menschen in Deutschland 3,3 Mio. ( $80 \%$ ) überwiegend von ihren Angehörigen zu Hause versorgt [18].

Auch ohne die pandemiebedingten Einschränkungen sind pflegende Angehörige mit vielen Herausforderungen konfrontiert, die häufig mit körperlichen und psychischen Beeinträchtigungen einhergehen [15, 17, 19]. Erste Onlinebe- fragungen belegen eine Zunahme der Belastungen pflegender Angehöriger in Deutschland im Zuge der Coronapandemie $[8,16]$. Eine Verschlechterung des eigenen Gesundheitszustandes wurde in einer Onlinebefragung von der Hälfte der pflegenden Angehörigen angegeben [16]. Die in dieser Untersuchung befragten Angehörigen (<67 Jahre) pflegten Personen aller Altersgruppen ( $\geq 60$ Jahre: $45 \%$ ). Für ältere Pflegende kommt erschwerend hinzu, dass sie neben den Gepflegten häufig 
selbst ein erhöhtes Risiko (z.B. aufgrund eigener Grunderkrankungen, Immunseneszenz) für schwere und tödliche COVID19-Verläufe haben [14, 21].

Zur Entlastung pflegender Angehöriger in der Coronapandemie wurden durch das Bundeskabinett Akuthilfen beschlossen. Hierzu gehören erweiterte Leistungen bei Pflegezeit und kurzzeitiger Arbeitsverhinderung sowie flexiblere Gestaltungsmöglichkeiten bei der Pflege- und Familienpflegezeit [5]. Für ein besseres Verständnis, welche Entlastungen pflegende Angehörige darüber hinaus benötigen, sind differenzierte Untersuchungen der Veränderungen ihrer Pflegesituationen und ihres Belastungserlebens notwendig.

Vor diesem Hintergrund wurden Teilnehmende einer Interventionsstudie für hochbelastete pflegende Angehörige im Rahmen einer Zusatzbefragung zu Auswirkungen der Coronapandemie auf ihre Pflege- und Lebenssituation befragt. Die von ihnen gepflegten Personen waren mindestens 60 Jahre alt. Ergänzend zu den bisherigen Onlinebefragungen sollten mittels einer Offlineerhebung auch pflegende Angehörige mit geringem bzw. ohne Internetzugang oder-nutzung erreicht werden. Zudem sollten potenzielle Prädiktoren des Belastungserlebens der pflegenden Angehörigen und der Pflegebedürftigen untersucht werden.

\section{Material und Methoden}

\section{Stichprobe}

Die vorliegende Befragung erfolgte als ergänzende Erhebung im Rahmen einer randomisierten kontrollierten Interventionsstudie zu einem gestuften Beratungsansatz für hochbelastete pflegende Angehörige [13]. Als Intervention wurde ein strukturiertes Problemlösen für pflegende Angehörige im Rahmen einer Pflegeberatung ( $§ 7$ a SGB XI) mit einer nachfolgenden optionalen telefonischen psychotherapeutischen Unterstützung kombiniert und mit einer Kontrollgruppe (Regelversorgung) verglichen.

Eingeschlossen wurden pflegende Angehörige, die jemanden pflegen, der a) mindestens 60 Jahre alt ist, b) einen Pflegegrad hat und $c$ ) bei der Allgemeinen Ortskrankenkasse (AOK) Baden-
Württemberg oder AOK Bayern versichert ist. Die Pflegenden mussten d) mindestens 18 Jahre alt sein, e) durchschnittlich mindestens 1,5h/Tag bzw. 10,5 h/Woche Pflege/Betreuung für die gepflegte Person leisten, f) ein positives Belastungsscreening haben (mindestens 2 der 3 folgenden Kriterien lagen vor: 1) pflegebedingte Einschränkung der psychischen/körperlichen Gesundheit, 2) Einsamkeit, 3) Pflegebelastung). Rekrutiert wurden die Probanden über die kooperierenden Pflegekassen der Gepflegten. Die Ausschlusskriterien sowie ausführliche Informationen zur Hauptstudie können dem Studienprotokoll entnommen werden [13].

Den bis Ende April 2020 in die Hauptstudie eingeschlossenen $(n=235)$ pflegenden Angehörigen wurde ein Fragebogen zum Belastungserleben während der Coronapandemie postalisch zugesandt. Davon sendeten 165 Personen (70,2\%) den Fragebogen im Zeitraum April bis Anfang Juni 2020 an das Studienteam zurück. Die Probanden dieser zusätzlichen Untersuchung unterscheiden sich hinsichtlich der Baseline-Daten nicht von den Pflegenden, die nicht an der vorliegenden Befragung teilgenommen haben (Alter $(p=0,5)$, Geschlecht $(p=0,21)$, Pflegedauer $(p=0,58)$, Verhältnis zum Gepflegten $(p=0,38)$, depressive Symptomatik (Allgemeine Depressionsskala [ADS]; [9]; $p=0,89$ ) und körperliche Beschwerden (Gießener Beschwerdebogen [GBB-24]; [2]; $p=0,33)$ ).

\section{Messinstrumente}

Die Erfassung des Belastungserlebens in der Coronapandemie erfolgte über die Entwicklung von themenspezifischen Items durch die Arbeitsgruppe. Auswirkungen der Pandemie auf die Pflegebelastung, pandemiebedingte Ängste und 18 konkrete Auswirkungen auf die Lebens- und Pflegesituation wurden mittels einer 5-stufigen Antwortskala erfasst (z. B. „Ich fühle mich einsamer als vor der Coronapandemie"; 0: überhaupt nicht; 4: äußerst). Weiterhin wurden Veränderungen der Inanspruchnahme formeller Unterstützungsangebote erhoben. Zudem sollten Pflegende aktuelle zwischenmenschliche Spannungen in ihrem Haushalt auf einer 10-stufigen Skala einschätzen (0: über- haupt keine Spannungen; 10: sehr hohe Spannungen). Ergänzend wurden mittels 3 offener Fragen Auswirkungen der Pandemie auf den Pflegealltag, ausgelöste Sorgen sowie Wünsche der Pflegenden in Bezug auf die Pflege während der Pandemie erfragt. Abschließend wurden mittels 4 dichotomer (1: ja; 0: nein) Items eine aktuelle Coronaerkrankung, -symptome, -testungen und Quarantänepflicht sowie das Arbeiten im Homeoffice erfasst.

Als relevante Prädiktoren für das Belastungserleben wurden neben dem Geschlecht (1: Mann, 2: Frau) das Alter und der Angehörigenstatus (0: Partnerln, 1: (Schwieger-)Kind) der pflegenden Angehörigen sowie der Pflegegrad, das Vorliegen einer Demenzerkrankung bei den Erkrankten (0: nein, 1: ja) und die Nutzung von Tagespflege, Pflegedienst und ambulanter häuslicher Betreuung vor der Coronapandemie (0: nein, 1 : ja) berücksichtigt.

\section{Analysen}

Die deskriptiven Analysen erfolgten mit SPSS 25 (IBM, Armonk, NY, USA). Antworten der offenen Fragen wurden mittels qualitativer Inhaltsanalyse [11] analysiert. Dabei wurden für jede Frage induktiv von der Zweitautorin und einer wissenschaftlichen Mitarbeiterin ein Kodiersystem entwickelt. Beide kodierten die Texte unabhängig voneinander; anschließend wurden die Kategorienauswahl überprüft, Unterschiede nach der Hälfte der kodierten Aussagen diskutiert und das Kategoriensystem entsprechend angepasst. Kodiereinheiten bildeten die stichpunktartigen Aussagen der Pflegenden. Für jede der 3 offenen Fragen wurden die Häufigkeiten der Kategorien sowie die Übereinstimmung der Kodierungen berechnet. Die Reliabilität der Kategoriensysteme „Auswirkungen auf den Pflegealltag" (Krippendorffs $a=0,86$ ), "Sorgen und Ängste" (Krippendorffs $a=0,89$ ) und "Wünsche" (Krippendorffs $a=0,91$ ) ist gegeben [10]. Die Kategorien sind den $\bullet$ Tab. 1, 2 und 3 zu entnehmen.

Für das Belastungserleben der Pflegenden (Item „Inwieweit hat sich Ihre Pflegebelastung durch die Coronapandemie erhöht") und die Einschätzung des Belastungserlebens der Erkrankten 
Tab. 1 Auswirkungen der Coronapandemie auf den Pflegealltag (219 Aussagen von $n=128$ )

\begin{tabular}{|l|l|l|}
\hline Kategorie & Häufigkeit & $(\%)$ \\
\hline Ausfall/Reduktion von Pflegedienstleistungen & 52 & $(23,7)$ \\
\hline Weniger Zeit für sich & 27 & $(12,3)$ \\
\hline Weniger Sozialkontakte der Gepflegten & 24 & $(11)$ \\
\hline Erhöhte Angst/Sorgen der Pflegenden & 18 & $(8,2)$ \\
\hline Gesteigerte Hygienemaßnahmen & 17 & $(7,8)$ \\
\hline Alltag ist aufwendiger & 16 & $(7,3)$ \\
\hline Wenig Verständnis für Situation durch Gepflegte & 13 & $(5,9)$ \\
\hline Weniger Freizeitaktivitäten der Gepflegten & 10 & $(4,6)$ \\
\hline Weniger Unterstützung durch Familie, Freunde & 9 & $(4,1)$ \\
\hline Weniger (Körper)Kontakt zw. Angehörigen und Gepflegten & 7 & $(3,2)$ \\
\hline Ausfall/Verschiebung wichtiger Untersuchungen & 7 & $(3,2)$ \\
\hline Durch Kurzarbeit/Homeoffice mehr Zeit bei Gepflegten & 4 & $(1,8)$ \\
\hline Sonstiges & 15 & $(6,8)$ \\
\hline
\end{tabular}

\begin{tabular}{|l|l|l|}
\hline Tab. 2 Sorgen/Ängste, ausgelöst durch die Coronapandemie (188 Aussagen von $n=128)$ \\
\hline Kategorie: Angst um/vor ... & Häufigkeit & $(\%)$ \\
\hline Sicherstellung der Pflege & 29 & $(15,4)$ \\
\hline Gesundheit der Gepflegten & 27 & $(14,4)$ \\
\hline Eigene Gesundheit & 23 & $(12,2)$ \\
\hline Finanzielle Existenz & 18 & $(9,6)$ \\
\hline Zukunft allgemein/Wirtschaft & 16 & $(8,5)$ \\
\hline Vereinsamung, Isolation & 15 & $(8,0)$ \\
\hline Gepflegte anzustecken & 13 & $(6,9)$ \\
\hline Familie (Gesundheit, Finanzen, Kontaktverbot) & 12 & $(6,4)$ \\
\hline Einschränkung der Freiheit/Grundrechte & 8 & $(4,3)$ \\
\hline Weniger Zeit für Erholung/Angst vor Überlastung & 8 & $(4,3)$ \\
\hline Sonstiges & 19 & $(10,1)$ \\
\hline
\end{tabular}

\begin{tabular}{|l|l|l|}
\hline Tab. 3 Wünsche aufgrund der Coronapandemie in Bezug aufPflege (154 Aussagen von $n=125)$ \\
\hline Kategorie & Häufigkeit & $(\%)$ \\
\hline Mehr/Wiederaufnahme der Unterstützung & 60 & $(39,0)$ \\
\hline Wiederherstellung der Normalität & 22 & $(14,3)$ \\
\hline Mehr soziale Kontakte/Freizeit & 17 & $(11,0)$ \\
\hline Gesund bleiben & 12 & $(7,8)$ \\
\hline Bessere Ausstattung (Schutzkleidung, Desinfektionsmittel, Tests etc.) & 12 & $(7,8)$ \\
\hline Mehr Wertschätzung häuslicher Pflege & 6 & $(3,9)$ \\
\hline Impfstoffentwicklung & 6 & $(3,9)$ \\
\hline Besserer Umgang mit Situation in Pflegeeinrichtungen & 4 & $(2,6)$ \\
\hline Sonstiges & 15 & $(9,7)$ \\
\hline
\end{tabular}

durch die Pflegenden (Item „Mein Angehöriger leidet in seinem Alltag unter der Coronapandemie") wurden getrennt hierarchische Regressionen mit 2 Blöcken durchgeführt (Block 1: Alter, Geschlecht, Beziehungsstatus; Block 2: Pflegegrad, Demenzerkrankung, Nutzung von Tagespflege, Pflegedienst und ambulanter häuslicher Betreuung). Durch den blockweisen Einschluss sollte die separierte

\section{Ergebnisse}

\section{Stichprobe}

Die pflegenden Angehörigen ( $n=165)$ waren im Mittel 59,8 $( \pm 9,69)$ Jahre alt und zumeist weiblich (87,3\%). Die mittlere Pflegedauer betrug $5,6( \pm 4,54)$ Jahre. Weitere Charakteristika der pflegenden Angehörigen sowie die 5 häufigsten Erkrankungen der Gepflegten sind 0 Tab. 4 zu entnehmen.

\section{Erleben und Belastungen während der Coronapandemie}

Ein Viertel der Pflegenden (26,3\%) gibt starke oder sehr starke Ängste vor einer eigenen Ansteckung mit COVID-19 an. Die Hälfte befürchtet, dass sich die gepflegte Person anstecken könnte (50,3\%). Zudem berichten $46,6 \%$ der Pflegenden deutliche Auswirkungen der Pandemie auf den Pflegealltag. Im Rahmen der offenen Fragen werden als Auswirkungen auf den Pflegealltag am häufigsten der Ausfall oder die Reduktion erhaltener Pflegedienstleistungen $(23,7 \%)$, weniger Zeit für sich selbst $(12,3 \%)$ sowie weniger Sozialkontakte der Gepflegten (11\%) genannt (0 Tab. 1).

Zitat ID 84:

Entlastung durch Sozialstation ist weggefallen, momentan keine Haushaltshilfe; Belastung (Psyche) wird für mich größer, da größere Isolation, zunehmende Unzufriedenheit der Pflegeperson, da jetzt zusätzlich „eingesperrt".

Die Mehrheit (78\%) der Pflegenden nimmt eine Erhöhung der Pflegebelastung wahr, wobei die Hälfte $(50,6 \%)$ sogar eine starke oder sehr starke Zunahme berichtet. Jeder 4. pflegenden Person $(24,2 \%)$ fällt es ziemlich bis äußerst schwer, die Pflege ihrer Angehörigen in gewohnter Weise aufrechtzuerhalten.

Ein Drittel $(33,2 \%)$ erhält deutlich weniger oder keine Hilfe mehr durch Familie oder Freunde. Auch die Inanspruchnahme professioneller Unterstützungsangebote verringerte sich: 97,6\% der Gepflegten, die bislang Tagespflege in Anspruch genommen hatten (24,8\%), können diese seit Corona nicht mehr nutzen. Einen ambulanten Pflegedienst nutzten vorher $25 \%$ der Pflegenden, von diesen nutzen 10\% diesen seltener und $24,3 \%$ nicht mehr. Ei- 


\begin{tabular}{|c|c|c|c|c|}
\hline & $M(S D)$ & Spanne & $n$ & $\%$ \\
\hline \multicolumn{5}{|l|}{ Pflegende Angehörige $(N=165)$} \\
\hline Alter (Jahre) & $59,8(9,69)$ & $33-83$ & - & - \\
\hline Geschlecht (Frauen) & - & - & 144 & 78,3 \\
\hline \multicolumn{5}{|l|}{ Beziehung zur gepflegten Person } \\
\hline Partnerln & - & - & 67 & 40,7 \\
\hline Kind & - & - & 89 & 53,9 \\
\hline Andere & - & - & 9 & 5,4 \\
\hline $\begin{array}{l}\text { Zusammenleben mit der gepfleg- } \\
\text { ten Person }\end{array}$ & - & - & 95 & 57,6 \\
\hline Pflegedauer (Jahre) & $5,6(4,54)$ & $<1-33$ & - & - \\
\hline Depressive Symptome (ADS) & $22,2(0,31)$ & - & - & - \\
\hline Körperbeschwerden (GBB-24) & $30,7(16,40)$ & - & - & - \\
\hline \multicolumn{5}{|l|}{ Gepflegte Personen $(N=165)$} \\
\hline Alter (Jahre) & $79,4(8,74)$ & $60-95$ & - & - \\
\hline Geschlecht (Frauen) & - & - & 87 & 52,7 \\
\hline \multicolumn{5}{|l|}{ Pflegegrad } \\
\hline 1 & - & - & - & 3,7 \\
\hline 2 & - & - & - & 35,4 \\
\hline 3 & - & - & - & 32,3 \\
\hline 4 & - & - & - & 18,9 \\
\hline 5 & - & - & - & 9,8 \\
\hline \multicolumn{5}{|l|}{ Erkrankung } \\
\hline Geheinschränkungen & - & - & - & 59,5 \\
\hline Demenz & - & - & - & 49,7 \\
\hline Inkontinenz & - & - & - & 41,7 \\
\hline Herzschwäche & - & - & - & 36,2 \\
\hline Depression & - & - & - & 31,3 \\
\hline
\end{tabular}

sogar äußerst stark unter den Einschränkungen.

Die aktuelle zwischenmenschliche Spannung im Haushalt wird von $66,5 \%$ der Pflegenden als mittel bis sehr hoch eingeschätzt. Weitere Sorgen und Ängste, welche durch die Pandemie ausgelöst wurden, belasten 37,9\% der Pflegenden deutlich. Die im Rahmen der offenen Frage am häufigsten geschilderten weiteren Ängste sind Sorgen um die Sicherstellung der Pflege (15,4\%), der Gesundheit der Gepflegten $(14,4 \%)$ und der eigenen Gesundheit (12,2\%) (• Tab. 2). Die Ergebnisse der Befragung sind in $\mathbf{0}$ Tab. $\mathbf{6}$ und 7 zusammengefasst.

Zitat ID 30:

Ich kann meine Mutter nicht mehr richtig versorgen, wenn ich krank werde.

Im Rahmen der offenen Frage nach Wünschen in Bezug auf die aktuelle Situation geben pflegende Angehörige am häufigsten den Wunsch nach einer Wiederaufnahme/Zunahme von Unterstützung (39\%), Wiederherstellung der Normalität $(14,3 \%)$ und Zunahme sozialer Kontakte (11\%) an (•Tab. 3).

Zitat ID 369:

Bessere Unterstützung bei den durch die Coronapandemie verursachten mentalen Leiden

Tab. 5 Inanspruchnahme formeller Unterstützungsangebote während der Coronapandemie \begin{tabular}{|l|l|l|}
\hline Unterstützungsangebot & Inanspruch- & Veränderungen seit Pandemiebeginn
\end{tabular}

\begin{tabular}{|l|l|l|l|l|l|}
\hline & & $\begin{array}{l}\text { Inanspruch- } \\
\text { nahme vor Pan- } \\
\text { demiebeginn }\end{array}$ & \multicolumn{4}{|l|}{ Verănderungen seit Pandemiebeginn } \\
\cline { 2 - 6 } & $n$ & $\begin{array}{l}\text { Keine Ver- } \\
\text { änderung }\end{array}$ & Seltener & $\begin{array}{l}\text { Keine Nut- } \\
\text { zung mehr }\end{array}$ & Häufiger \\
\cline { 3 - 6 } & $n(\%)$ & $n(\%)$ & $n(\%)$ & $n(\%)$ \\
\hline $\begin{array}{l}\text { Tagespflege/ } \\
\text { Betreuungsgruppe }\end{array}$ & 41 & $1(2,4)$ & - & $40(97,6)$ & - \\
\hline Pflegedienst & 70 & $45(64,3)$ & $7(10,0)$ & $17(24,3)$ & $1(1,4)$ \\
\hline Haushaltshilfe & 54 & $20(37,0)$ & $7(13,0)$ & $27(50,0)$ & - \\
\hline $\begin{array}{l}\text { Ambulante häusliche } \\
\text { Betreuung }\end{array}$ & 23 & $3(13,0)$ & $4(17,4)$ & $12(52,2)$ & $1(4,3)$ \\
\hline Ehrenamtliche Betreuung & 14 & - & $2(14,3)$ & $12(85,7)$ & - \\
\hline Angehörigengruppe & 19 & $4(21,1)$ & $2(10,5)$ & $10(52,6)$ & $3(15,8)$ \\
\hline
\end{tabular}

ne Haushaltshilfe nahmen vorher $32,7 \%$ in Anspruch, von diesen nehmen $13 \%$ seltener und $50 \%$ keine Haushaltshilfe mehr in Anspruch. Veränderungen der Inanspruchnahme formeller Unterstützungsangebote von Personen, welche diese vor der Pandemie genutzt hatten, sind 0 Tab. 5 zu entnehmen.
Zudem schildern 52,5\% der Pflegenden, dass ihre Erholungsmöglichkeiten deutlich eingeschränkt sind und sie sich auch deutlich einsamer als vor der Pandemie fühlen (31,3\%). Nach Einschätzung der Angehörigen leiden mehr als jeder 3. Pflegebedürftige $(37,5 \%)$ ziemlich oder der zu Pflegenden (Vereinsamung aufgrund von Kontaktverbot/Depression).

\section{Prädiktoren des Belastungserlebens}

Bezüglich des Belastungserlebens der Pflegenden konnte keines der beiden Regressionsmodelle ein signifikantes Niveau erreichen (Modell 1: $p=0,06$; Modell 2: $p=0,14$ ), auch wenn die Ergebnisse für das Geschlecht der Pflegenden im Block 1 auf einen positiven Zusammenhang zum Belastungserleben der Pflegenden hindeuten $(\beta=0,17, p=0,04)$. Für das durch die Pflegenden eingeschätzte Belastungserleben der Erkrankten konnte durch beide Blöcke $9,5 \%$ der Varianz erklärt werden (Regressionsmodell 2 mit beiden Blöcken: $p<0,01)$, wobei Alter $(\beta=-0,25$, $p<0,05)$, Pflegegrad $(\beta=-0,23, p<0,01)$ und die Nutzung des Pflegedienstes vor der Pandemie $(\beta=0,25, p<0,01)$ einen signifikanten Zusammenhang mit dem 
Tab. 6 Auswirkungen der Coronapandemie in Prozent $(n=165)$

\begin{tabular}{|l|l|l|l|l|l|}
\hline Item & $\begin{array}{l}\text { Überhaupt } \\
\text { nicht }\end{array}$ & $\begin{array}{l}\text { Ein } \\
\text { wenig }\end{array}$ & $\begin{array}{l}\text { Mittel- } \\
\text { mäßig }\end{array}$ & $\begin{array}{l}\text { Ziem- } \\
\text { lich }\end{array}$ & Äußst \\
\hline $\begin{array}{l}\text { Auswirkungen der Pandemie auf Pflegeall- } \\
\text { tag }\end{array}$ & 9,3 & 20,5 & 23,6 & 32,9 & 13,7 \\
\hline Erhöhung der Pflegebelastung & 5,5 & 16,5 & 27,4 & 36 & 14,6 \\
\hline Angst sich anzustecken & 24,5 & 27 & 22,1 & 19,6 & 6,7 \\
\hline Angst vor Ansteckung der Gepflegten & 11 & 17,8 & 20,9 & 30,1 & 20,2 \\
\hline $\begin{array}{l}\text { Anspannung beim Gedanken, das Haus zu } \\
\text { verlassen }\end{array}$ & 17,6 & 28,5 & 22,4 & 17,6 & 13,9 \\
\hline Einschränkung von Erholungsmöglichkeiten & 12,8 & 16,5 & 18,5 & 30,5 & 22 \\
\hline Einschränkung der Freiheit & 8 & 18,5 & 22,8 & 32,7 & 17,9 \\
\hline Anstieg der Einsamkeit & 22,7 & 25,8 & 20,2 & 20,9 & 10,4 \\
\hline $\begin{array}{l}\text { Reduktion/Wegfall der Hilfe von Familie/ } \\
\text { Freunden }\end{array}$ & 38,1 & 13,1 & 15,6 & 18,8 & 14,4 \\
\hline $\begin{array}{l}\text { Aufrechterhalten der Pflege/Betreuung in } \\
\text { gewohnter Weise fällt schwer }\end{array}$ & 29,1 & 24,2 & 22,4 & 20,6 & 3,6 \\
\hline $\begin{array}{l}\text { Wissen über Beratungsmöglichkeiten bei } \\
\text { Unterstützungsbedarf }\end{array}$ & 14,7 & 16,6 & 19 & 27,6 & 22,1 \\
\hline Auslösung weiterer Sorgen & 9,9 & 28 & 24,2 & 27,3 & 10,6 \\
\hline Angst der Gepflegten sich anzustecken & 46,9 & 17,9 & 13,6 & 13,6 & 8 \\
\hline Gepflegte leiden unter Einschränkungen & 27,6 & 17,2 & 17,8 & 21,5 & 16 \\
\hline $\begin{array}{l}\text { Gepflegte leiden im Alltag unter der Pande- } \\
\text { mie }\end{array}$ & 30,9 & 23,5 & 18,5 & 17,3 & 9,9 \\
\hline $\begin{array}{l}\text { Gepflegte scheinen aus Sicht der Pflegen- } \\
\text { den keine Auswirkungen zu bemerken }\end{array}$ & 29,6 & 25,3 & 21 & 11,1 & 13 \\
\hline
\end{tabular}

\begin{tabular}{|l|l|l|}
\hline Tab. 7 Auswirkungen auf den Alltag $(n=165)$ & Nein (\%) & Ja (\%) \\
\hline Item & 87,8 & 12,2 \\
\hline Absage eines Rehaaufenthalts & 87,8 & 12,2 \\
\hline Absage einer Krankenhausbehandlung & 89,9 & 10,1 \\
\hline Absage einer Krankenhausbehandlung der Gepflegten & 93,3 & Positiv: 0,6 \\
\cline { 2 - 3 } Bisherige Testung auf das Coronavirus & & Negativ: 6,1 \\
\hline COVID-19-Symptome in letzten 7 Tagen & 100 & 0 \\
\hline Aktuelle Quarantäne & 98,8 & 1,2 \\
\hline Berufstätige: Homeoffice & 76,5 & 23,5 \\
\hline
\end{tabular}

eingeschätzten Belastungserleben aufwiesen.

\section{Diskussion}

Die vorliegende Studie bestätigt in eindrücklicher Weise die hohe Zusatzbelastung von ohnehin schon belasteten pflegenden Angehörigen. Obwohl die Befragten selten von einer Coronavirusinfektion oder Quarantäne betroffen waren, hat die Pandemie weitreichende Folgen für ihre Lebens- und Pflegesituation. So hatte ein Viertel der Pflegenden starke oder sehr starke Angst, sich selbst mit COVID-19 zu infizieren, und die Hälfte auch davor, dass sich die gepflegte Person infizieren könn- te. Die Ängste vor einer Infektion sind in dieser Stichprobe etwas häufiger vertreten als in der Studie des Zentrums für Qualität in der Pflege (ZQP) und der Charité (April/Mai 2020), in der ebenfalls auch ältere pflegende Angehörige ( $\geq 70: 21 \%$ ) online befragt wurden [8]. Hier berichteten $20 \%$ der Pflegenden starke oder sehr starke Ängste vor einer eigenen Infektion sowie $40 \%$ bezüglich einer Infektion der Gepflegten. Im Rahmen dieser Studie gab ein Viertel der Pflegenden an, dass die aktuelle Pflegesituation sie überfordere, was für $4 \%$ „voll und ganz" und für $21 \%$ „eher zutraf". Sorge, die Pflege nicht mehr zu schaffen, gab ebenfalls ein Viertel der Befragten an, wobei dies für $4 \%$ „voll und ganz" und für $19 \%$ „eher zutraf“. In der vorliegenden Befragung mit belasteten Angehörigen wurden die pandemiebedingten Auswirkungen in ähnlicher Weise oder noch schwerwiegender wahrgenommen. So gab ein Viertel der Pflegenden an, dass die Aufrechterhaltung der Pflege in der gewohnten Art und Weise ihnen „ziemlich" oder "äußerst" schwerfalle; ein weiteres Viertel stimmte dieser Aussage zumindest teilweise („mittelmäßig“) zu.

Die Aufrechterhaltung der häuslichen Pflege wurde insbesondere durch Wegfall oder Reduzierung professioneller und informeller Unterstützungsangebote erschwert. Ein Drittel der Pflegenden der vorliegenden Studie musste die Pflege mit deutlich weniger Hilfe durch Familie oder Freunde bewältigen. Dabei zeigen Studien, dass besonders informelle Unterstützung eine der wichtigsten Ressourcen pflegender Angehöriger bei der Bewältigung von Pflege ist [3].

Zusätzlich sind formelle Unterstützungsangebote zur Entlastung weggefallen, was sich in der vorliegenden Studie in besonderen Maße zeigt. Während nahezu alle Pflegenden nicht mehr die Tagespflege oder Betreuungsgruppen nutzten und etwa ein Drittel der Pflegenden weniger oder keine Leistungen mehr durch ihren ambulanten Pflegedienst erhielt, gaben in der ZQP und Charité-Studie $85 \%$ bzw. $20 \%$ der Befragten einen entsprechenden Rückgang oder Ausfall von Leistungen der Tagespflege und ambulanten Pflege an. Budnick et al. [4] konnten in weiterführenden Analysen der Studie des ZQP und der Charité zeigen, dass insbesondere Angehörige, die professionelle Unterstützungsangebote nicht mehr nutzen konnten, eine Verschlechterung der Pflegesituation wahrnahmen und mehr negative Gefühle angaben. Anders als in der Studie von Budnick et al. [4] zeigte das Vorliegen einer Demenzerkrankung der Pflegebedürftigen in der aktuellen Studie keinen Zusammenhang zur Belastung der Pflegenden. So ließen sich in der vorliegenden Studie keine eindeutigen Prädiktoren des Belastungserlebens der Pflegenden im Zusammenhang mit der Coronapandemie finden, was komplexere Zusammenhänge verschiedener Faktoren und auch die Bedeutsamkeit von psychischen Faktoren (z.B. Coping-Strategien) vermuten lässt. 
Allerdings wiesen der Pflegegrad der Erkrankten und das Alter der Pflegenden einen negativen Zusammenhang und die vorherige Nutzung des Pflegedienstes einen positiven Zusammenhang zum Belastungserleben der Pflegebedürftigen auf, eingeschätzt durch die Pflegenden. Aus Sicht der Angehörigen scheinen Pflegebedürftige, welche einen niedrigeren Pflegegrad haben und vor der Pandemie einen Pflegedienst genutzt hatten, stärker unter den pandemiebedingten Einschränkungen zu leiden. Anzunehmen ist, dass die pandemiebedingten Einschränkungen für Erkrankte mit einer höheren Mobilität eine stärke Begrenzung ihrer Erlebensund Bewegungsräume darstellen und der Wegfall des Pflegedienstes als Minderung der pflegerischen Versorgung wahrgenommen wird.

Zur psychosozialen Belastung der Pflegenden tragen auch die soziale Isolation, erlebte Einsamkeit der Gepflegten und pflegenden Angehörigen bei, was sich in den qualitativen und quantitativen $\mathrm{Er}$ gebnissen deutlich widerspiegelt. Zudem sind Pflegebedürftige unabhängig von der Grunderkrankung (sofern noch mobil) durch Quarantänemaßnahmen in ihrer körperlichen Aktivität eingeschränkt. Mangelnde Bewegung beeinflusst wiederum zahlreiche weitere Gesundheitsparameter in negativer Weise und kann dadurch die Pflege zusätzlich erschweren [1].

Besonders der Wegfall von Unterstützungsmöglichkeiten beeinträchtigt die Erholungs- und Regenerationsmöglichkeiten der Pflegenden. Hierdurch steigt das oft bereits vor der Pandemie vorhandene Risiko einer Überlastung und Überforderung, was sich wiederum negativ auf die Pflegequalität auswirken kann. Erschwerend ist, dass auch Pflegeberatungen ( $\$ 7$ a SGB XI), Beratungsbesuche ( $\S 37.3$ SGB XI) und Begutachtungen ( $§ 18$ SGB XI) im Rahmen von Hausbesuchen ausgesetzt wurden und sich dadurch die Beratungsmöglichkeiten deutlich verschlechtert haben.

Bei einer längerfristigen Überlastungssituation ist von vermehrten körperlichen und psychischen Langzeitfolgen bei pflegenden Angehörigen auszugehen. Deswegen sind neben staatlichen Akuthilfen weitere Unterstützungsangebote notwendig, die zur Verbesserung der Versorgung und sozialen Teilhabe der Pflegebedürftigen sowie der pflegenden Angehörigen beitragen und Langzeitfolgen verhindern oder abmildern [7].

Wie den Wünschen der befragten Angehörigen zu entnehmen war, wurden die Verfügbarkeit von Schutzmaterialien (Masken, Desinfektionsmitteln, Tests etc.) und die Schulung in deren Anwendung als zentral für den Erhalt von informeller und formeller Unterstützung angesehen. Darüber hinaus sind niederschwellige telefon- und internetbasierte Angebote der Beratung (z.B. zu verfügbaren Hilfen und Unterstützungsangeboten) und Schulung (z.B. Pflegekurse) durch kompetentes Personal zur Unterstützung der Pflegenden notwendig. Zur Vermeidung psychischer Langzeitbelastungen bei Pflegenden haben sich individuelle psychologische Interventionen, basierend auf der kognitiven Verhaltenstherapie, als besonders wirksam erwiesen [6]. Der Zugang zu entsprechenden evaluierten psychologischen telefonischen und internetbasierten Interventionen sollte hochbelasteten pflegenden Angehörigen ermöglicht werden [12, 20].

Stärken der vorliegenden Studie sind die telefonische Rekrutierung über die Krankenkasse der Gepflegten und die schriftliche Befragung, wodurch auch Pflegende ohne Internetzugang oder -nutzung eingeschlossen werden konnten. Zudem geben die offenen Fragen und die qualitativen Analysen einen differenzierten Einblick, inwieweit sich Lebensund Pflegesituationen pflegender Angehöriger durch die Pandemie verändert haben.

Die Ergebnisse verdeutlichen eindrücklich die Verstärkung der ohnehin belastenden Situation pflegender Angehöriger während der Coronapandemie. Zur Bewältigung der komplexen zusätzlichen Herausforderungen benötigen pflegende Angehörige Unterstützungsangebote, die ihre aktuelle Problem- und Bedarfslage berücksichtigen und dieser gerecht werden.

\section{Fazit}

- Hochbelastete pflegende Angehörige nehmen nahezu alle eine pandemiebedingte Zunahme der Pflegebelastung wahr.
- Der Großteil der Pflegenden hat Angst, sich selbst oder die gepflegte Person mit SARS-CoV-2 zu infizieren.

- Die Mehrzahl der Pflegenden leidet unter Einsamkeit, fehlenden Erholungsmöglichkeiten und einer Zunahme von Sorgen.

- Niedrigschwellig verfügbare, gut in den Pflegealltag integrierbare Unterstützungsangebote sind unabdingbar und bestehende online Angebote (u. a. AOK Pflegecoach, Onlinepflegekurse) müssen umfänglich bekannt gemacht werden.

\section{Korrespondenzadresse}

\section{Christina Theurer}

Institut der Psychologie, Abteilung KlinischPsychologische Intervention, Friedrich-SchillerUniversität Jena

Semmelweißstr. 12, 07743 Jena, Deutschland christina.theurer@uni-jena.de

Danksagung. Wir danken allen pflegenden Angehörigen für ihre Studienteilnahme. Zudem bedanken wir uns für die Zusammenarbeit und Unterstützung durch die ReDiCare (deutsch: BerTA) Studiengruppe. Hierzu zählen Grit Stößel und Maximilian Diepold (beide Friedrich Schiller Universität Jena), Prof. Dr. Dietrich Rothenbacher, Dr. Gisela Büchele, Jennifer Müller, Martin Rehm (alle Universität Ulm), Prof. Christian Ernst und Helene Dick (Universität Hohenheim), Prof. Dr. Thomas Heidenreich und Prof. Dr. Astrid Elsbernd (beide Hochschule Esslingen), Dr. Ana Babac (AOK Bayern) und Frau Maria Gonzalez Medina (AOK Baden-Württemberg).

Förderung. Die Befragung erfolgte im Rahmen einer durch das Bundesministerium für Bildung und Forschung geförderten Interventionsstudie (Förderkennzeichen 01GL1702).

Funding. Open Access funding enabled and organized by Projekt DEAL.

\section{Einhaltung ethischer Richtlinien}

Interessenkonflikt. C. Theurer, D. Rother, K. Pfeiffer und G. Wilz geben an, dass kein Interessenkonflikt besteht.

Alle im vorliegenden Manuskript beschriebenen Untersuchungen am Menschen wurden mit Zustimmung der zuständigen Ethikkommission, im Einklang mit nationalem Recht sowie gemäß der Deklaration von Helsinki von 1975 (in der aktuellen, überarbeiteten Fassung) durchgeführt. Von allen beteiligten Studienteilnehmenden liegt eine Einverständniserklärung vor.

Open Access. Dieser Artikel wird unter der Creative 
Commons Namensnennung 4.0 International Lizenz veröffentlicht, welche die Nutzung, Vervielfältigung, Bearbeitung, Verbreitung und Wiedergabe in jeglichem Medium und Format erlaubt, sofern Sie den/die ursprünglichen Autor(en) und die Quelle ordnungsgemäß nennen, einen Link zur Creative Commons Lizenz beifügen und angeben, ob Änderungen vorgenommen wurden.

Die in diesem Artikel enthaltenen Bilder und sonstiges Drittmaterial unterliegen ebenfalls der genannten Creative Commons Lizenz, sofern sich aus der Abbildungslegende nichts anderes ergibt. Sofern das betreffende Material nicht unter der genannten Creative Commons Lizenz steht und die betreffende Handlung nicht nach gesetzlichen Vorschriften erlaubt ist, ist für die oben aufgeführten Weiterverwendungen des $\mathrm{Ma}$ terials die Einwilligung des jeweiligen Rechteinhabers einzuholen.

Weitere Details zur Lizenz entnehmen Sie bitte der Lizenzinformation auf http://creativecommons.org/ licenses/by/4.0/deed.de.

\section{Literatur}

1. Aubertin-Leheudre M, Rolland Y (2020) The importance of physical activity to care for frail older adults during the COVID-19 pandemic. J Am Med Dir Assoc 21:973-976

2. Braehler E, Hinz A, Scheer J (2008) GBB-24. Der Gießener Beschwerdebogen (GBB). Huber, Bern

3. Brügger S, Jaquier A, Sottas B (2016) Belastungserleben und Coping-Strategien pflegender Angehöriger Perspektive der Angehörigen. Z Gerontol Geriat 2:138-142

4. Budnick A, Hering C, Eggert S et al (2021) Informal caregiversduring the COVID-19pandemicperceive additional burden: findings from an ad-hoc survey in Germany. BMC Health Serv Res. https://doi.org/ 10.1186/s12913-021-06359-7

5. Bundesministerium für Familie, Senioren, Frauen und Jugend (2021) Akuthilfen für pflegende Angehörige sind verlängert. https://www.bmfsfj. de/bmfsfj/aktuelles/alle-meldungen/akuthilfenfuer-pflegende-angehoerige-sind-verlaengert160232.Zugegriffen: 1. Apr. 2021

6. Cheng ST, Li KK, Losada A et al (2020) The effectiveness of nonpharmacological interventions for informal dementia caregivers: an updated systematic review and meta-analysis. Psychol Aging 35:55-77

7. Deutsche Gesellschaft für Gerontologie und Geriatrie (2020) Gemeinsames Statement der Sektionen Sozial- und Verhaltenswissenschaftliche Gerontologie (III) und Soziologie und Altenarbeit (IV) der DGGG. https://www.dggg-online.de/ fileadmin/aktuelles/covid-19/20200510_DGGG Statement_Sektionen_III_IV_Menschen_mit Pflegebedarf.pdf.Zugegriffen: 31. März 2021

8. Eggert S, Teubner C, Budnick A et al (2020) Pflegende Angehörige in der COVID-19-Krise. Ergebnisse einer bundesweiten Befragung. www.zqp.de/wp-content/uploads/ZQP-AnalyseAngehörigeCOVID19.pdfZugegriffen: 31. März 2021

9. Hautzinger M, Bailer M, Hofmeister D et al (2012) Allgemeine Depressions Skala. Manual. Hogrefe, Göttingen, Germany

10. Hayes AF, Krippendorff K (2007) Answering the call for a standard reliability measure for coding data. Commun Methods Meas 1:77-89

\section{Burden experienced by caregiving relatives during the corona pandemic}

Background: Family caregivers are faced with a variety of challenges and burdens. Recent online studies showed that these burdens have increased even more during the corona pandemic. Here we conducted a low-threshold written survey to assess psychological distress in highly burdened family caregivers during the corona pandemic in Germany.

Method: Both quantitative and qualitative analyses were conducted within the context of a tiered counselling intervention study for highly burdened family caregivers $(N=165)$ to assess their experience of burden, anxieties, worries and desires regarding care.

Results: A quarter of all caregivers (26\%) reported high fear of becoming infected with SARS-CoV-2 and 50\% feared their care recipient could get infected. Almost half of all caregivers reported (47\%) that corona had a high impact on the care situation and increased the burden of caregiving (51\%). A loss of professional support and less time for themselves were reported most often. The desire for additional support was frequently expressed. The level of care, the age of the caregiver and the previous use of care services were found to be predictors of the psychological distress experienced by care recipients.

Conclusion: The results illustrate an increased burden related to caregiving during the corona pandemic. In order to cope with this complex additional burden, there is an even higher need for support to improve care and social participation for both care recipients and caregivers during the pandemic.

\section{Keywords}

Informal care · Caregiver burden · Psychosocial burden · SARS-CoV-2 · COVID-19

11. Mayring $P$ (2004) Qualitative content analysis. In: Flick U, von KardorfE, Steinke I (Hrsg) A companion to qualitative research. SAGE, London, S266-269

12. Meichsner F, Theurer C, Wilz G (2019) Acceptance and treatment effects of an internet-delivered cognitive-behavioral intervention for family caregivers of people with dementia: a randomizedcontrolled trial. JClin Psychol 75(4):594-613

13. Pfeiffer K, Theurer C, Buchele G et al (2021) Relieving distressed caregivers (ReDiCare study): study protocol of a randomized pragmatic trial. BMC Geriatr. https://doi.org/10.1186/s12877020-01941-w

14. Robert Koch Institut (2020) Informationen und Hilfestellungen für Personen mit einem höheren Risiko für einen schweren COVID-19-Krankheitsverlauf. https://www.rki.de/DE/Content/InfAZ/ N/Neuartiges_Coronavirus/Risikogruppen.html. Zugegriffen:31. März 2021

15. Romero-Moreno R, Marquez-Gonzalez M, Mausbach BT et al (2012) Variables modulating depression in dementia caregivers: a longitudinal study. Int Psychogeriatr 24:1316-1324

16. Rothgang H, Wolf-Ostermann K (2020) Zur Situation der häuslichen Pflege in Deutschland während der Corona-Pandemie. Ergebnisse einer OnlineBefragung von informellen Pflegepersonen im erwerbsfähigen Alter. Schnellbericht. https://www. socium.uni-bremen.de/uploads/Schnellbericht_ Befragung_pflegender_Angehoriger_-_print. pdf. Zugegriffen: 31. März 2021

17. Sittler MC, Wilz G (2020) Quality of sleep in caregiving relatives of people with dementia: investigation of the prevalence and possible predictors. ZGerontol Geriat 53:303-309

18. Statistisches Bundesamt (2020) Pressemitteilung Nr. 507 vom 15. Dezember 2020. https://www.
destatis.de/DE/Presse/Pressemitteilungen/2020/ 12/PD20_507_224.html. Zugegriffen: 31. März 2021

19. Tramonti F, Bonfiglio L, Bongioanni P et al (2019) Caregiver burden and family functioning in different neurological diseases. Psychol Health Med 24:27-34

20. Wilz G, Reder M, Meichsner F et al (2018) The Tele.TAnDem intervention: telephone-based CBT for family caregivers of people with dementia. Gerontologist 58:e118-e129

21. Zhou F, Yu T, Du R (2020) Clinical course and risk factors formortality of adultinpatients with COVID19 in Wuhan, China: a retrospective cohort study (vol 395, pg 1054, 2020). Lancet 395:1038-1038 